\title{
Health by Numbers? Exploring the practice and experience of datafied health
}

\author{
Gavin J.D. Smith, Research School of Social Sciences, ANU \\ Ben Vonthethoff, The Regional Australia Institute
}

\begin{abstract}
The widespread availability of portable sensing devices has given rise to growing numbers of people voluntarily self-tracking their daily experiences through the medium of digital data. At the extreme end of this trend is the 'Quantified Self' movement. This collective uses sensor-enabled tech to extensively map aspects of their personal lives, before sharing procedural insights at community 'show and tell' events. A key aim of the group is to better understand imperceptible bodily processes, especially those influencing health states, as they are materialised through the datafied body. Despite the growth in those mobilising digital data for health management, little is known about the subjective meanings that are ascribed to self-monitoring practices. This paper explores how self-trackers conceptualise the data they generate, and how exteriorised bodily interiorities mediate impressions of embodiment. We suggest that the availability of self-tracked data has initiated interesting new relationships between data-subjects and their objectified bodies, dynamics that impact on how bodies are experienced and inhabited. We show how bodily intuition is being outsourced to, if not displaced by, the medium of 'unbodied' data. It is this objectivated facility that is increasingly used to orientate behavioural decisions as they relate to bodily maintenance.
\end{abstract}

Keywords: Self-tracking, subjectivity, embodiment, datafied health, intuition, knowledge,

\section{Introduction}

If you want to replace the vagaries of intuition with something more reliable, you first need to gather data (Gary Wolf, co-founder of QS). ${ }^{\text {i }}$

Today's world is awash with digital data: data that both structures and represents aspects of human experience, from birth and social relationships to self-identity and death. As people interact with sensor-enabled technologies that are increasingly mobile, networked, and attached to the body_-such as smartphones and fitbits—flows of personal data relating to embodied processes and behaviours are created, captured, and circulated. These media devices have afforded new opportunities for individuals to monitor, map, and visualise everyday events such as eating, running, and expenditure and to correlate these with distinct determinants. Although most data is created unconsciously_as people interact with, and through, ambient sensor technologies - there is a growing number of people interested in actively logging their physiologies, movements, moods, and experiences in order to detect previously unseen bodily processes and behavioural habits. This social practice is popularly referred to as 'self-tracking', and it involves the extensive monitoring and measuring of bodily performance and activities via the medium of digital data in order to chart how corporeality relates to external factors.

Underpinning practices of self-tracking is the idea that data-driven knowledge can provide instruction for those seeking to exercise greater control over their lives. 
Dalton and Thatcher (2014) contend that the progressive reduction of social life into flows of data requires social scientists to explore how such media 'open up' and 'close off' particular social experiences and practices. As self-tracked digital data promises to reveal and remember more about our inner dynamics than we ourselves can consciously register via the body's sensorial faculties, it becomes imperative to examine the phenomenological dimensions of self-tracking on understandings of embodiment. This is both a conceptual and empirical task. Contemporary health-care provides an illuminative context in which to analyse this issue: to consider how processes of health provision and consumption are being transformed by practices of self-tracking and the production of digital data.

This paper looks at the Quantified Self community (QS hereafter), a globally dispersed group of self-trackers who creatively render selected bodily actions into data as a means to garner insights that can inform processes of behavioural refinement. As a large and diverse contingent deliberately 'prosuming'-that is, producing and consuming - data about their daily practices and biotic states, the QS represent an ideal, if exceptional, sample of people who seek to manage health conditions through the medium of digital data. Notwithstanding the significant growth in those mobilising technologies and digital data for these purposes, little is known about the subjective meanings that are ascribed to such cultural practices. In particular, there are limited empirical studies that explore the interrelationship between practices of self-monitoring, flows of data, and modes of subjectivity.

We examine how self-trackers in-or on the margins of - the QS community conceptualise and narrate the data they generate, and how the exteriorised data derived from bodily processes mediates their experiences of embodiment. We suggest that the availability of self-tracked data has established interesting new relationships between data-subjects and their objectified bodies, dynamics that have a significant effect on how individuals inhabit their bodies. We point to the way in which the bodily intuition of self-trackers is being 'outsourced' to, if not displaced by, the medium of 'unbodied' (i.e. from the body, but not of the body) data; it is this objectivated facility (Berger and Luckmann, 1966, pp. 60-61) that is increasingly used as an intuitive device for orientating behavioural decisions as they relate to bodily maintenance.

\section{The reflexive subject and self-tracking practices}

Self-tracking repertoires need to be situated within what Beck et al. (1994) call, reflexive modernisation, a context in which subjects become increasingly individualised, introspective, and responsible for the project of crafting their own selfidentities. Reflexive modernisation is also characterised by a ubiquity of risks which the sovereign state is unable to eradicate, and the consequent need for cosmopolites to manage their own risk loading via the internalisation of expert advice, the exercise of prudent behaviours, and the uptake of available technologies. With these circumstances in mind, it is not difficult to comprehend why some adopt a 'technoutopian' perspective of self-tracking, perceiving it as a core aspect of preventive medicine (Swan, 2009; Smarr, 2012). While there are indubitable upsides of datafied and devolved health initiatives, these accounts tend to exaggerate the actual capabilities of the technologies, especially by overstating their potential to administer patient empowerment and tangible health benefits. They often fail to acknowledge the 'social, ethical and political implications' of self-monitoring data in the realm of personal healthcare (Lupton, 2013a, p. 257). 
Deborah Lupton (2013a, p. 258), for instance, accentuates the 'digitally engaged patient' notion to describe the way in which lay people are 'encouraged to take an active role in producing and consuming information about health' by using commercial smartphone apps. In this model, patients are expected to assume responsibility for their own health needs via the regimes of personal visibility they enact, representing an archipelago of the medical gaze from the clinic to a more diffuse and plural 'techno-gaze' that permeates any social space mediated through the networked prism of the data sharing device. Lupton (2012, pp. 234-5) argues that these interventions provide individuals with a platform to exteriorise internal bodily dynamics as virtual maps, while simultaneously offering to third-party onlookers 'an unprecedented opportunity to monitor and measure individuals' health-related habits in a variety of milieu'.

The implications of these transformations in contemporary healthcare delivery are not insignificant. For example, Martin French and Gavin Smith (2013) note that encouraging patients to adopt commercially-driven tracking devices and infrastructures as a mode of preventive healthcare reflects the neoliberal concern to marketise and outsource the costs and responsibilities of health provisions, while devolving the burden of wellbeing onto the self-monitoring individual. They point to the implicit assumptions incorporated in these aspirations which tend to presuppose publics being universally endowed with the resources, skills, and literacy needed to access and operate these technologies. In addition, Gavin Smith (2016) has shown how data-subjects are often neither aware nor in exclusive proprietary possession of the proxy flows of 'data exhaust' that emanate from their bodies. Moreover, Lupton (2015a) has demonstrated that the experience of tracking sexual activity on a number of available smartphone apps is bifurcated as a result of gender codes. For example, those apps designed for female use tend to be framed in terms of the reproductive risks associated with sexual transmitted diseases, while those for men typically focus on enriching sexual stamina and performance. Such platforms are never free of politics and norms, and instead merely reify prevailing gender biases and hierarchies. Significant questions arise regarding how self-tracked data is exploited by different parties for programs of health management (e.g. self-trackers, state agencies, corporations, and insurance actuaries), and how this leakage of information into the bordering 'technosphere' impacts on a data subject's consequent social experiences and life chances (Smith, 2016).

Notwithstanding its potential to render data-subjects susceptible to the gaze of an unknown agency, Nafus and Sherman (2014) suggest that self-tracking represents a form of 'soft resistance' to the monolithic health promotion rhetorics endorsed by governmental agencies and commercial corporations. Such discourses tend to reduce the complexity of health experiences and needs to an idealised-and thus generalised-paradigm. For example, personalised notions of 'what is healthy for me?' are particularly common mantras among self-trackers and these individualised self-articulations function to challenge the aggregated definitions of 'healthiness' favoured in medicalised discourses of health, and reflected in the architectures and designs of a number of fitness and self-tracking apps. As such, their concept of 'soft resistance' elucidates how self-trackers conform to broader cultural scripts of maintaining and inhabiting a healthy mind-body, but also shape how this trope is practically achieved at the personal level (Nafus and Sherman, 2014, p. 1785). Yet Lupton (2014b) contends that actual opportunities for soft resistance are limited only to specific modes of voluntary self-tracking. The idea therefore is not necessarily applicable to involuntary self-tracking practices such as the measurement of worker 
productivity by employers or when probationary services subject offenders to mandatory tagging programs. In these examples where the data harvested serves ends and interests above and beyond those of the monitored and objectified subject, possibilities for defiance are severely restricted.

Minna Ruckenstein (2014) found that individuals engaged in self-tracking as a means to make a substantive change in how they organised and performed their personal regimes of health and fitness, especially by being able to retain and analyse better records of bodily physiology and habits over time. She (ibid, p. 78) highlights how the virtualised diagram of the body/self visualised through the medium of data intensified self-tracking desires and practices, creating a tangible materialised entity for inspection, measurement, and refinement. Such 'techno-bodied' figures provided self-trackers with opportunities to chart bodily and emotional experiences over time and in relation to specific contexts, catalysts, and events, and to compare these against the social graphs of others. Ruckenstein's focus on motives for self-tracking reveals the nuanced types of social meaning ascribed to experiences of this practice. Certain self-trackers moralised devices like the heart rate monitor as 'external motivators' for actioning healthier behaviours; their presence in or on the body, and their capacity to network in an external audience through data transmissions, acting as a disciplinary lever that mediated dietary and exercise decisions.

Research by Hortensius et al. (2012) exposes the ambiguity of self-tracking practices, demonstrating how being the object/subject of monitoring can elicit feelings of confidence, certainty, and wholesomeness, while simultaneously generating experiences of anxiety and fragility when the data display unexpected or undesirable results. Yet Nafus and Sherman (2014, p. 1789) argue that self-trackers 'in no way cede authority' to the supposed objectivity of these actants. Nor do they attribute extra validity to their outputs simply because of their quantitative, numeric, and computational attributes. Instead, datafied figures of the body/self act as dialectical mediums through which people negotiate meanings.

Our research offers a nuanced conclusion to that of Nafus and Sherman, demonstrating how the datafied figure of the body/self that emerges from selftracking practices creates new sensor-driven frameworks for data-subjects to locate bodily intuition and insight. These frameworks function to turn the self-tracker's attention away from the signals and sensations of the embodied sensorium toward a technical sensing apparatus that privileges algorithmic analytics. Although the pursuit of self-knowledge and self-optimisation fundamentally depends on the organic body as the principal 'object of information', the means of sensemaking is increasingly outsourced to and performed by auxiliary codifying mechanisms that are adjudged to encompass greater degrees of validity and reliability. This raises important sociological questions pertaining to contemporary experiences of embodiment, especially the displacement of 'feeling representations' to data-driven sensemaking infrastructures that become primary oracles of the objectivated body.

\section{Methods}

This study adopted an online ethnographic approach to analyse 'naturally occurring' qualitative material posted on the official QS website, in terms of presented narratives, statistics, and iconography. We were keen to interrogate the language selftrackers use during their 'show and tell' confessions and the sentiments shared between presenter and audience, as well as visual representations of and responses to self-tracking practices/experiences. These accounts provided details pertinent to our 
interest in how and why people engage with and through digitised data, what data means to them, and how it was leveraged for personal outcomes.

The sample was comprised of 30 videos, and featured the reflections of 5 female and 25 male self-trackers from predominantly professional and tech backgrounds in the US. Most of the excerpts were approximately 7-8 minutes in duration. Each of these were fully transcribed and then coded in accordance with inductive (i.e. from the data) and deductive (i.e. from the literature) thematic categories. The approach adopted was similar to that of Choe et al. (2014) who purposively sampled 52 videos for analysis between January 2012 and April 2013. These authors used a two-part exclusion criteria requiring: 1) that the speaker talked about their own data practices; and 2) that the presentation contained a legible data visualisation for additional context. We copied this rubric, but applied it only to the most recent videos uploaded to the site when the research was conducted-i.e. from August 2014 to July 2015. This allowed for an entirely new dataset to be analysed. The excerpts presented below were selected on the basis that they revealed important aspects of the interplay between self-tracked data, embodiment, and narratives of the self.

Online research can raise difficult ethical considerations, in terms of distinguishing what is public, from what is private, data, deciding whether informed consent need be attained from the research 'participants' given the lack of spatial and temporal proximity, and determining whether a researcher is obliged to disclose her identity. For the purpose of this preliminary research, the data utilised predominantly originates from the voluntary communicative acts of a 'networked public' (boyd, 2014: 5), and it is therefore treated in this spirit as a public asset and resource: as amenable for interrogation. Two semi-structured qualitative interviews were also conducted with individuals not affiliated with the QS, but who were enthusiastic users of self-tracking devices and personal analytic infrastructures. This was to produce a richer account of self-data relations, and to probe questions and clarify issues not obtainable from the analysis of pre-recorded and thus circumscribed videos.

\section{The social life of self-tracking}

The QS is an international collaboration of designers and users of self-tracking tools and mediums. Founded in 2007 by Gary Wolf and Kevin Kelly and comprising a worldwide network of approximately 43,000 self-trackers, QS organises regular conventions for distributed self-trackers to transfer the knowledge they gleaned from their self-monitoring practices and experiences of digital data. They use a dedicated website to post information on self-tracking techniques, practices, and events, and to embed the monitory activities of individual self-trackers within a broader community framework. As Kelly (2012) succinctly puts it, QS is principally about the pursuit of 'self-knowledge through numbers.' It is about individuals learning to read and inhabit their bodies in new ways through the mediums of data and audience feedback, but also to use such insight to reform entrenched habits and to enrich their life circumstances.

In contrast to concerns within scholarly circles about the surveillant capacities of sensor devices (Andrejevic \& Gates, 2014), Kelly and Wolf highlight the participatory and empowering scope of self-tracking and data sharing practices. Prioritising the virtues of the entrepreneurial and reflexive self, self-trackers believe that data sharing repertoires provide a means to indirectly access opaque somatic processes and the unconscious self, and thereby to produce a blueprint of physiology 
and subjectivity that can be worked on and optimised. They hold and reify the belief that there is an affective layer of experience that can be detected and accessed through the medium of data alone. From this perspective, data functions as a window and conduit to interiority, and provides the self-tracker with a data-driven lens through which they can more autonomously manage their lives.

Nafus and Sherman (2014, p. 1787) found QS meetups to be 'relatively coherent across sites', each encompassing a similar format and script. The theatrical 'show and tell' presentations are a customary feature of QS meetups, and these confessional monologues initiate a solidarity among self-trackers, in terms of presenters explaining to a susceptive audience how and why technologies were designed and selected, what the self-tracking program sought to reveal and what it actually exposed, and how it acted as a catalyst for further self-analysis and behavioural modification. As Sharon and Zandbergen (2016, p. 10) note,

Standing on stage, self-trackers speak about painful episodes in their lives (depression, divorce, disease); they expose their dreams, their diary entries and their meditation practices, and they reveal minutiae about their physical ailments and their struggles with weight and mental well-being. Far from an aggregation of data-obsessed narcissists, then, what one witnesses here is closer to a confessional community, where numbers are used to 'confess' intimate details of personal lives to others.

It would be easy to surmise that the hyper-individualised self-tracker identity eclipses that of being a fellow 'QSelfer' member/participant. But we would argue in contrast that, for the reasons alluded to by Sharon and Zandbergen above, the QS audience-along with the 'dispersed audience' embedded in the implanted datatransmitting mechanisms-provide motivation not necessarily for initiating, but certainly for persevering with, self-tracking practices. For some, the impetus to selftrack is socially-driven and exceeds the exclusive quest for self-knowledge, reflecting desires instead to narrate personal experiences and stories in a public forum via the 'companion' medium of their data. The audience functions to validate the personal struggles and technical gadgetry of self-trackers, it supports potentially discrediting presentations of self, and affirms that the obsessive tracking of bodily experiences is normal and desirable. They help institute a collective consciousness valorising practices of self-exposure, and situate data-subjects in a dual role as subjects and agents of surveillance.

Of course, we realise there is a spectrum of affiliations to the organisation and we do not wish to enter into debates here as to whether or not the QS represents a comparable community for all its contributors. But we do want to accentuate the social life of data, the fact that digital devices and media infrastructures function to convert aspects of personal experience into a textual format that facilitates modes of dialogical exchange. In Kelly's (2009) view, data sharing is 'the mildest form of socialism', actuating experiences of comradeship and companionship, and serving 'as the foundation for higher levels of communal engagement'. For Lupton (2013b, p. 29), these behaviours are 'central to the web 2.0 age, in which sharing of data and other forms of content is valorised.' As she asserts, when individual trackers engage online or meet up in person, 'the quantified self becomes the quantified community' (ibid, p. 28).

\section{The addictiveness of data}


In an era of liquid modernity, the body becomes progressively characterised as porous and without limits (Bauman, 2005, p. 93). This absence of frontier is epitomised, Bauman (2000, pp. 77-8) contends, by the cultural ideal of 'fitness' - a term that does not denote a particular bodily condition, but rather its potential for continuous refinement. The notion of fitness is open-ended and is personified by the maxim, 'however fit your body is-you could always make it fitter' (Bauman, 2005, p. 93). This way of conceptualising the body frames it as an ongoing project that demands the investment of attentiveness and labour, and the consumption of enrichment goods and services. Self-tracking practices are intrinsically bound to such logics. They are the outcome of technological development, discourses of self-reflexiveness, and efforts to optimise one's own life by continuously fine-tuning mind-body togetherness (Lupton, 2013a; 2014a). Data is key to such transformations in self-determination.

Ruckenstein (2014, p. 73) found participants in her research regarding selftracking as a 'catalyst for change'. Using data to orientate one's behaviour in this way evokes Foucault's (1988) seminal conceptualisation of technologies of the self. Technologies of the self are mechanisms, ideas, and practices, from self-help manuals to medicines, that constitute the ways people engage in caring for the self (i.e. in 'subjectivating' themselves), in becoming aware of its latent and incomplete character and being attuned to its various developmental needs: in the interminable pursuit of engineering a stable and productive sense of self. Such techniques typically conform to expert (of the political and commercial sort) discourses pertaining to the subjective management of wellbeing. More often than not, they operate in highly normative ways as a mode of power that brings the errant or flawed self into line with governmental expectations and social conventions. Mutually related discourses of self-imperfection and self-improvement are common in the QS community, and they are specifically prevalent in the narratives expressed by those seeking to enhance their health states through the objectivating character of data. The data is positioned as providing the instruction, discipline, and impetus needed to enact a lifestyle intervention. Data is in this sense a medium of subjectivation. Its production reflects the internalisation of cultural obsessions with 'finding the self' with a view to managing it better via data-driven modes of knowing.

Data is construed as a conduit for making meaningful alterations to daily routines and habits that are deemed harmful or irrational, and for maximising lived experiences of the body: to develop its capacities to think, feel, communicate, seduce, and act. In this way, practices of self-tracking and data accretion intersect with desires to enhance personal circumstances and to exert control over social trajectory. For example, Kevin, in his mid-fifties and suffering from Parkinson's disease exhibits a subjectivated narrative of data as a means of amelioration. He speaks of his wish to improve his overall quality of life through mutual practices of self-tracking and datafication:

[The] main thing is to optimise productivity and quality of life...maximise productivity, type faster, work faster, run around the office faster, reduce stress...optimise routines...improve sleep results.

Likewise, Anand, who is in his mid-twenties and has developed his own 'gyroscope' app to aggregate the various data traces from his everyday actions into a virtual 'event matrix’ that records/visualises every moment of his life, told a QS audience: 
I wanted to fix my vitamin D levels, run faster, lower my heart rate, I wanted to be more productive... basically just become superman.

Anand highlights the importance of data in his pursuit of self-development, stating that 'actually tracking the stuff is really, really important, and the numbers are what kind of tell you what to do'. This technophilic account is intriguing for it reveals how data are subject to fetishisation, attributed with a veracity and transformative agency that comes, in a dialectical fashion, to wield a significant influence on how dataproviders understand themselves and orientate their behaviours. Anand goes onto note, 'one thing I realised was that I got sick a few times, and I looked back on my logs, and my heart rates were way higher, like in the hundreds ... So that was an interesting benchmark of figuring out, hey I'm sick, just from the numbers.' His data do not entirely override sensations deriving from his sensory faculties, but instead add a horizon of instruction to organise his quests for the acquisition of the appropriate biocapital that will boost his physiological performances, and thus supply him with a sense of achievement and validation.

Discourses of personal responsibility were also prevalent motives and outcomes in many of the other QS presentations. Kouris, in his late-thirties and tracking his weight and sleep trends, pointed out that, 'it's important to find out what the data tells you, because then it's your choice to actually do something about it'. This kind of verbalisation attests how subjective attentiveness is outsourced to the data representations to the point where forms of embodied knowledge are marginalised as unhelpful or untrustworthy, especially bodily signals, sensations, and intuitiveness. In the search for data-driven answers and directions, embodied consciousness is temporarily transferred from the interiorities and sensations of the body, to instead focus on their exteriorised representation as mediated visualisations.

In the context of QS, practices of self-tracking have a disciplinary and subjectivating quality. A key aspect of meetup events is the confessional admission where presenters disclose to a networked public the personal problem they sought to objectivate and address through data-driven analytics, the moment of epiphany when a detrimental habit or factor became apparent, and the responses they consequently mobilised. This ritual operates to reify the perceived need to engage in voluntary modes of self-surveillance while enrolling participants into conformist behaviours that pertain to dominant discourses of self-imperfection, metricisation, and improvement through the guise of data-driven visibility and self-directed autonomy. In Anand's case - where he deliberately made his personal data available for public scrutiny-a key inspiration was to receive 'motivational [read disciplinary] feedback' from a virtual audience: 'I built this just for myself ... but I decided to put it up on the internet. There was no privacy, nothing is secret, everything I do is out there.' He goes onto describe how exposing his self-tracked data impacted on his consequent behaviour:

It changed my behaviour a lot. One thing I realised was I started showing off and running way longer...people also started emailing me telling me I'm a wuss and I should run more, which was really motivating. The idea of sharing everything turned out to be a really positive experience for me.

The networked audience became a source of stimulus for Anand, not only through its projected gaze, but also through its mediated interaction with him, inciting his desire to push himself harder and further on each run and thus materialise his 
aspiration of enhanced fitness and vitality. Similarly, when describing her tracking of type-II diabetes, Alice told the Bay Area meetup group how she aspired to make her health data-points available to her primary care provider and family, as she felt the anticipated solidarities provided by her support team would keep her more focused on the 'burdensome' task of managing her illness. Alice's case attests how the existence of data enables the exercise of discipline to be outsourced from the territories of the self to an external audience, albeit in her case a familial one:

I'd like to be able to share this with my family, especially as I get older. They might want to know what's going on in my life especially when they're not nearby... And making sure they're helping me to keep honest and keep on top of what's going on.

As Bauman (2005, p. 17) contends, the elusive search for a stable identity in a liquid world means we have little choice but to look 'inside of ourselves'. Both in the context of the QS community in general and the interviewees in particular, selftrackers expressed a strong desire to increase the supply of data relating to their bodies. The will for greater self-knowledge and bodily enlightenment was framed around an inherent dissatisfaction with-and distrust of - the sensing, feeling, and intuitive organic body as a bounded instrument of illumination and as a vehicle of change. It was grounded instead in the belief that an increase in the quantity and quality of data could improve embodied wellness. This doctrinal perception encourages the self-tracker to transcend the perceived limitations of biological forms of sensing. The paradoxical process of looking beyond - while simultaneously looking at-the body creates the preference for implementing ambient recording devices that can objectivate corporeality in its natural and non-contrived state, and in an unfiltered way that does not invalidate the objectivity of the results. Such multi-layered and multi-mediated looking is founded on ontological and epistemological assumptions. It implicitly construes devices as untainted bearers of truth and bodies as pure 'objects of information', as providing stable (i.e. datafiable) reference points for the sourcing of the required knowledge. There is a tacit appreciation that the nature of interiorised affects cannot be derived from direct consciousness alone and the resultant difficulties of approaching the object of investigation (the body/self) as a subjectivated inhabitant. They must instead be exteriorised through the medium of digital data.

Kate, a university student who engaged in sleep-tracking to help manage a chronic condition, described how she wanted to measure variables other than sleep for greater contextual awareness of illness trigger factors, a task she felt her conscious mind alone could not achieve: 'I would definitely prefer to be tracking mood during the day; that would actually be useful... it would just be one more layer to have. And you can never have enough data'. As Whitson (2013, p.175) notes, 'quantification of the self allows us to replace the holes in our memories and the vagaries of our intuition' with the apparent objectivity and perspicacity of data-based knowledge, a techno-driven movement that makes instinctual powers of the body-mind progressively more peripheral and obsolescent as sources of enlightenment, awareness, edification, and truth. In this deterministic turn to processes of externalisation and objectivation, knowledge of the body/self is only validated if it appears outside of the body/self, especially in the form of a multiplex data visualisation.

Bauman (2005, p.80) argues that liquid modernity is defined by the elusive pursuit of fulfilment, with seduction located more within the 'promise of satisfaction' 
than the 'experience of satisfaction' itself. This is typified in the logic of consumer societies where immediate contentment is subordinated by promises of eternal happiness (ibid, p. 83). Device-sensed data is seen to satisfy our desires to 'know' more about ourselves than what the body (and its biological sensors) alone can tender, yet it also functions to instil a normative and normalising perception that additional data will reveal further truths about selfhood, and its ecological interdependencies. This perception assents with a techno-utopic impression that self-understanding cannot be established via embodied introspection and meditation alone, requiring instead technological platforms that can excavate and present the surfaces and depths of the self without subjectifying biases. This renders the satisfaction of each datafied insight marginal to the potential of future data-driven self-knowledge, thus perpetuating an addictive striving for the creation of additional data from within. A number of QS speakers framed their striving for more data as an 'obsession', alluding to the fleeting satisfaction of visualised data that is prosumed and then quickly discarded as dated or obsolete, or considered only intelligible when amalgamated with supplementary data-points.

\section{Becoming with dialogical data}

Self-tracked data is used as a medium to generate stories for and about the body/self. But equally, the digital devices and data flows themselves become their own relaters and agents in what is a multi-authored text. The data-subject translates the data just as the data apps and outputs translate the actions of the self-tracker. In other words, datasubjects and their data proxies are mutually engaged in relational interplays and entanglements, they become with one another and mediate how each is presented in specific actor-networks (Smith, 2016). They function as interconnected parts of a body/self/data dialogue. As Klauser \& Albrechtslund (2014, p. 278) note, datafying the self 'is related to describing, signifying or interpreting the self in terms of material facts ... stories and metaphors ... From this perspective, quantification is about modes of presenting and structuring an account of the self, for example as a narrative configuration'. Lomborg and Frandsen (2015) research has shown how self-tracking as a social and cultural practice is fundamentally communicative and dialogical. Applying this framework to those participating in self-tracking can help us better understand how data is used in manifold ways to (a) inform on the body/self, (b) motivate and stimulate behaviours, and (c) construct social narratives of health. The self-tracker produces and defines the data as much as the data objectivates and subjectivates the body/self.

Commonly referred to as 'nudges'-i.e. vibrations when a certain number of steps are taken or audio alerts reminding device-bearers to exercise-the ability of devices to autonomously communicate to the user in real time, and structure their consequent actions, is becoming a common feature of self-tracking experiences (Lupton, 2015b, p. 1351). These nudges play a pivotal role in orchestrating-i.e. incentivising, pressurising, and automating - health-related behaviours in a 'technobody' symbiosis. Alice, for example, spoke proudly about her digital tracking system that exploits text messages to regularly remind her to measure her blood sugar level:

I had to track the highs and the lows, I had to be conscientious about the changes happening in my body... I can see a nice graph telling me I've been doing a good job or not doing a good job. 
Alice's self-tracking is emblematic of Lupton's (2013a) digitally engaged patient, where citizens are expected to use portable consumer technologies to serve biopolitical ends: to develop bodily expertise via seemingly discretional acts for leveraging on themselves to improve levels of desirability, vitality, and productivity. Her narrative also reveals the degree to which bodily attentiveness is devolved from embodied sentience to an external recording device that is not subject to bouts of amnesia, stress or intoxication. As a result, these technologies help cultivate a selfreflexive subject who is aware of the deficiencies of her/his consciousness and sensorium, and the corollary need to maintain the body via a repertoire of mandated behaviours that are prompted by the automated monitoring regimes of the embedded digital devices. Alice is not only adhering to dominant discourses-which shift the burden of healthcare onto the individual-but also reinforcing impressions of data as an unmediated and unfiltered source of truth, while consequently reifying perceptions of embodied sentience as a site of vulnerability. Yet for Alice, this shift was empowering: she felt reassured from being able to reclaim control over the management of a condition that had previously felt intractable.

In other cases, this affective dialogue between machine and person is not so straightforward. Presenting to the Bay Area QS meetup group, Paul, a male in his mid-fifties, describes his efforts to track what he calls his 'upsets' — which translate as intense sensations of stress. Rather than seek to directly address the complex social causes of his embodied distress, Paul's narration reveals the way that self-trackers sometimes opt to manage the expression of symptoms through an amalgam of technical means and techno-ideals. Using a wearable heart rate monitor to map when he entered into these intensities, the device would signal to him by emitting a flashing red light and an accompanying alert. As Paul describes:

When the machine told me I was upset, I would then talk into my audio recorder... so I could actually hear the way I got myself out of it [through breathing and calming utterances].

Paul initially doubted the reliability of the device's feedback loop, 'When I first started using it, when it would flash red I would sit there and debate the machine'you can't be right?!'-I started calling it 'freakback'. Yet by the end of his selftracking experience, Paul came to 'to accept the readings' as objective markers of his affective states. In this sense, Paul privileged the technology's reading of himself above his own intuitive cognition. In this way, human-machine relationalities can produce a contested dialogic, where human-based agency is confronted with machinebased actancy, a situation that can initiate struggles and 'feeling estrangement' that pivot on the perceived veracity of outputs. This entanglement of meanings, those generated by the mutual actions of subjects and objects, is a common one in discussions of digital data. A key concern is that with the increasing transfer of power to machine-based algorithms and the situation of such infrastructures within state and corporate fields, the embodied actors providing the data get excluded and alienated from the codifications that come to structure their everyday lives (Klauser \& Albrechtslund, 2014, p. 278).

While data are often presented as irrefutable, in many instances selfquantification operates in tandem with sensory experiences to produce emergent forms of social practice. As a result, data proxies can tender not only a digital artefact for objectivation, but also represent a medium to be negotiated with in processes of subjectivation (Lupton, 2012, p. 237). At times, their existence and content creates a 
disconnect between how someone should feel, and how they actually feel. In particular, certain self-trackers described how data-based measures failed to fully reflect the richness of phenomenological experience. Sara, a university student, described how her unbodied sleep-tracking app did not adequately reflect her embodied experiences of sleep and fatigue:

It tells you about that sleep for the night, and I don't think it always reflects how you feel... because you could have had a relaxed day, but the app would still say you've had a restless sleep.

Importantly, this ongoing negotiation between self-trackers and their data exhaust occurs even in cases where the data is interpreted as an accurate reflection of lived experience. For example, Kate's description of her sleep data reveals a deeply ambiguous relation, where the virtualised data-archive had the capacity to produce simultaneous goods and bads depending on what it conveyed and the resultant meaning she attributed to it:

You know, if it says 78\% sleep quality last night, that is pretty good for me ... It's kind of self-manipulative, I'll be like, 'oh I must have slept well, I'm going to have a good day today'.

And yet, for Kate, a restless sleep pattern was correlated with a higher risk of experiencing a seizure, a data-mediated reality that caused her considerable embodied distress. Moreover, there were other visualisations where despite the data indicating good sleeps, she suffered a seizure, and a breakdown in data-based trust ensued. As she describes: 'those are the kind of ... days where I'm more likely to not then use the app again.' In addition, she disclosed that she avoids looking at the data on the days following a seizure: 'I don't want to see it.' When asked to explain this choice, she described a feeling of data overload: 'just knowing pretty much... I don't want to deal with this.' As Kate negotiates with her data exhaust on a daily basis, she walks a fine line between what the proxy actually communicates, what she wants to see/hear, and what she then experiences on the bodily register.

Self-tracking with digital data, particularly in the case of QS, is a social and relational practice, as engaging with personal data becomes akin to a performative event (Lomborg \& Frandsen, 2015; Lupton, 2014a). As Lupton (2014a, p.9) contends, self-tracking is about not only the 'stories people tell themselves' through their data, but also the stories and types of selves they exhibit publically. In various presentations, QS speakers describe how they started self-tracking for health reasons and then started using the data to develop a more informed and coherent biography. For example, Lee, a serial lifelogger in his mid-thirties, aggregated data-points into an annual report visualisation which helped him escape the myopia of embodied memory and better narrate his year as a totality:

When you're creating a book for somebody else to look at, you analyse the data in a different way to how you would have done before ... I also started a narrative of my findings. This storytelling allowed me to translate the data into a better representation of the kind of person I was that year.

Similarly Cors, a visual arts student, converted a year's worth of self-portraits into a data visualisation as a way to track, but also present, his annual mental health report. 
For Cors, his exteriorised data allowed him to 'convey a personal story'-an interiority - which captured how his sensory experience of well-being was in fact ecologically related and dependent, and contingent on the status of his personal relationships and seasonal and environmental fluctuations. By creating a visually engaging graphic through which self-trackers like Cors communicate their experiences, 'self-knowledge and self-expression' is simultaneously achieved (Lupton, 2014a, p. 12). In these examples, self-tracking becomes not only a practice of self-monitoring and data-driven introspection, but also a mode of 'communicating dimensions of the self using visual or other material based on one's data' (Lupton 2013b, p.29). Therefore, the collective aspect of the QS is integral to bringing highly individualised people together through a shared medium, a factor which initiates a dialogue between the self, its data Other, and a networked audience.

\section{Conclusion}

The field of personal and public healthcare has become a terrain where networked digital sensors and techno-bodied subjects are engaged in mutual dialogues: relationalities that are fundamentally re-shaping conventional conceptualisations of embodiment, health, and illness. We have pointed to some of the complex interplays that are occurring in this field, interplays that incorporate the reciprocal interactivity between digitised devices and media infrastructures, modes of expertise, embodied subjects, and data flows and visualisations. What seems evident is that the unbodied biotexts visualised via self-tracking practices are increasingly mediating how dataproviders understand, experience, and inhabit the objectivated territories of their bodies. This opens up a new politics of the body which, as we have seen, can have empowering and disempowering dimensions, as data transmissions come to act as both enabling and constraining forces in the production of bodily and social relations. We have pointed to the way in which intuition is being 'outsourced' to, if not displaced by, the medium of unbodied data; it is this objectivated facility that is increasingly used as an intuitive device for orientating behavioural decisions as they relate to bodily maintenance. Key to understanding the implications of this emergent social practice is to initiate more participatory empirical studies that give prominence to the social meanings that data both convey and embody as referents and mediums of the subject.

\section{Acknowledgements}

We wish to thank the two reviewers and Deborah Lupton for their excellent and instructive comments on an earlier draft of this paper.

\section{References}

Andrejevic, M., \& Gates, K. (2014). Editorial big data surveillance: introduction. Surveillance \& Society, 12(2), 185-196.

Bauman, Z. (2000). Liquid Modernity. Cambridge: Polity Press.

Bauman, Z. (2005). Liquid Life. Cambridge: Polity Press.

Beck, U., Giddens, A., \& Lash, S. (1994). Reflexive Modernization: Politics, Tradition and Aesthetics in the Modern Social Order. Cambridge: Polity Press.

Berger, P., \& Luckmann, T. (1966). The Social Construction of Reality: A Treatise in the Sociology of Knowledge. London: Penguin. 
boyd, d. (2014). It's Complicated: The Social Lives of Networked Teens. New Haven, CT: Yale University Press.

Choe, E. K., Lee, N. B., Lee, B., Pratt, W., \& Kientz, J. A. (2014). Understanding Quantified-Selfers' Practices in Collecting and Exploring Personal Data. Proceedings of the 32nd Annual ACM Conference on Human Factors in Computing Systems (pp. 1143-1152). Toronto: ACM Press.

Dalton, C., \& Thatcher, J. (2014). What does a critical data studies look like, and why do we care? Seven points for a critical approach to 'big data'. Society and Space, http://societyandspace.com/material/commentaries/craig-dalton-and-jim-thatcherwhat-does-a-critical-data-studies-look-like-and-why-do-we-care-seven-pointsfor-a-critical-approach-to-big-data/, accessed 6/6/15.

Foucault, M. (1988). Technologies of the Self. In L. H. Martin, H. Gutman \& P. H. Hutton (Eds.), Technologies of the Self: A Seminar with Michel Foucault (pp. 1649). Amherst, MA: University of Massachusetts Press.

French, M., \& Smith, G. J. D. (2013). Health surveillance: new modes of monitoring bodies, populations, and polities. Critical Public Health, 23(4), 383-392. doi: 10.1080/09581596.2013.838210

Hortensius, J., Kars, M. C., Wierenga, W. S., Kleefstra, N., Bilo, H. J., \& van der Bijl J. J. (2012). Perspectives of patients with type 1 or insulin-treated type 2 diabetes on self-monitoring of blood glucose: a qualitative study. BMC Public Health, 12(1), 1-11.

Kelly, K. (2009). The new socialism. http://www.wired.co.uk/magazine/archive/2009/07/features/the-new-socialism, accessed 15/11/15.

Kelly, K. (2012). Kevin Kelly on the history and future of QS. http://quantifiedself.com/2012/10/kevin-kelly-on-the-history-and-future-of-qs/, accessed 16/11/15.

Klauser, F.R., \& Albrechtslund, A. (2014). From self-tracking to smart urban infrastructures: towards an interdisciplinary research agenda on big data. Surveillance and Society, 12(2), 273-286.

Lomborg, S., \& Frandsen, K. (2015). Self-tracking as communication. Information, Communication \& Society, 18, 1-13. doi: 10.1177/1368431006065717

Lupton, D. (2012). M-health and health promotion: the digital cyborg and surveillance society. Social Theory \& Health, 10(3), 229-244.

Lupton, D. (2013a). The digitally engaged patient: self-monitoring and self-care in the digital health era. Social Theory \& Health, 11(3), 256-270. doi:

10.1057/sth.2013.10

Lupton, D. (2013b). Understanding the Human Machine. IEEE Technology and Society Magazine, 32(4), 25-30. doi: 10.1109/MTS.2013.2286431

Lupton, D. (2014a). You are Your Data: Self-Tracking Practices and Concepts of Data. http://papers.ssrn.com/sol3/papers.cfm?abstract_id=2534211, accessed 10/11/15.

Lupton, D. (2014b). Self-tracking modes: reflexive self-monitoring and data practices. Paper given at the 'Imminent Citizenships: Personhood and Identity Politics in the Informatic Age' workshop, ANU, Canberra, http://papers.ssrn.com/sol3/papers.cfm?abstract_id=2483549, accessed 15/11/15.

Lupton, D. (2015a). Quantified sex: a critical analysis of sexual and reproductive selftracking using apps. Culture, Health \& Sexuality, 17(4), 440-453. doi: 10.1080/13691058.2014.920528 
Lupton, D. (2015b). Critical Perspectives on Digital Health Technologies. Sociology Compass, 8(12), 1344-1359. doi: 0.1111/soc4.12226

Nafus, D., \& Sherman, J. (2014). This One Does Not Go Up to 11: The Quantified Self Movement as an Alternative Big Data Practice. International Journal of Communication, 8(1), 1784-1794.

Ruckenstein, M. (2014). Visualized and interacted life: Personal analytics and engagements with data doubles, Societies, 4(1), 68-84. doi: 10.3390/soc4010068

Sharon, T., \& Zandbergen, D. (2016). From data fetishism to quantifying selves: Selftracking practices and the other values of data. New Media \& Society. doi: $10.1177 / 1461444816636090$

Smarr, L. (2012). Quantifying your body: A how-to guide from a systems biology perspective. Biotechnology Journal, 7(8), 980-991. doi: 10.1002/biot.201100495

Smith, G. J. D. (2016). Surveillance, data and embodiment: On the work of being watched. Body \& Society. doi:10.1177/1357034X15623622.

Swan, M. (2009). Emerging patient-driven health care models: an examination of health social networks, consumer personalized medicine and quantified selftracking. International Journal of Environmental Research and Public Health, 6(2), 492-525.

Whitson, J. R. (2013). Gaming the quantified self. Surveillance \& Society, 11(1/2), 163-176.

\footnotetext{
i Wolf, G. (2010). The data-driven life. http://www.nytimes.com/2010/05/02/magazine/02selfmeasurement-t.html, accessed 13/3/16.
} 